\title{
Surgical treatment of thoracic-abdominal aortic aneurysms
}

\author{
Marina Alves Jacintho de Mello*, Camila Alcalde Mazza and Edmo Atique Gabriel \\ Union of the Colleges of the Great Lakes- UNILAGO, Brazil
}

\begin{abstract}
Thoracoabdominal aortic aneurysm (TAAA) is characterized by enlargement of the aortic segment in the diaphragmatic crura and extends by a proximal and / or distal variable distance from that point. The main sources of morbidity during operative repair of TAAAs are multiple organ failure, paraplegia, and respiratory, cardiac, or renal complications. In this context, the recent introduction of endovascular techniques may extend TAAA repair indications to high-risk patients whose only alternative is now represented by better clinical therapy. The main objective of this article is to describe the surgical treatment of thoracoabdominal aortic aneurysms and the methodology used was the literature review. The long-term results of most thoracic or abdominal aortic aneurysm reconstructions are satisfactory. However, some patients require a reoperation for a separate aneurysm after the original aortic reconstruction.
\end{abstract}

\section{Introduction}

A thoracoabdominal aortic aneurysm (TAAA) is characterized by enlargement of the aortic segment in the diaphragmatic crura and extends by a proximal and / or distal variable distance from this point [1]. Historically, open surgical repair of TAAAs involves greater operative risk than repairs of aneurysms in other segments of the aorta. The main sources of morbidity during operative repair of TAAAs are multiple organ failure, paraplegia, and respiratory, cardiac, or renal complications. Experienced surgical centers now report lower mortality and morbidity rates for TAAA repair than before, largely because of the use of adjuncts to prevent target-organ ischemia [2].

The recent introduction of endovascular techniques may extend TAAA repair indications to high-risk patients whose only alternative is now represented by better clinical therapy [1]. The present article aimed to describe the surgical treatment of thoracoabdominal aortic aneurysms. The methodology used was the literature review.

\section{Development}

\section{Open repair}

The conventional treatment of TAAAs consists of replacing the graft with the reinsertion of the main branches of the aorta. Currently, a multimodal approach is used to reduce surgery trauma, maximizing organ protection. The surgical technique used, such as aneurysm extension, has a significant impact on the outcome of the procedure [3].

\section{Thoracoabdominal incision and aortic exposure}

The patient is positioned in the right lateral decubitus position (shoulders at $60^{\circ}$, pelvis at $30^{\circ}$ ). The upper portion of the thoracoabdominal incision is made through the 6th intercostal space; Anterolaterally, the incision curves smoothly as it crosses the costal margin, reducing the risk of tissue necrosis. The pleural space is inserted after the initiation of single right lung ventilation (Figure 1).

Paralysis of the left hemidiaphragm by its radial division to the aortic gap would contribute significantly to postoperative respiratory failure, so after the thoracoabdominal incision, a circumferential section of the diaphragm is routinely performed, sparing the phrenic

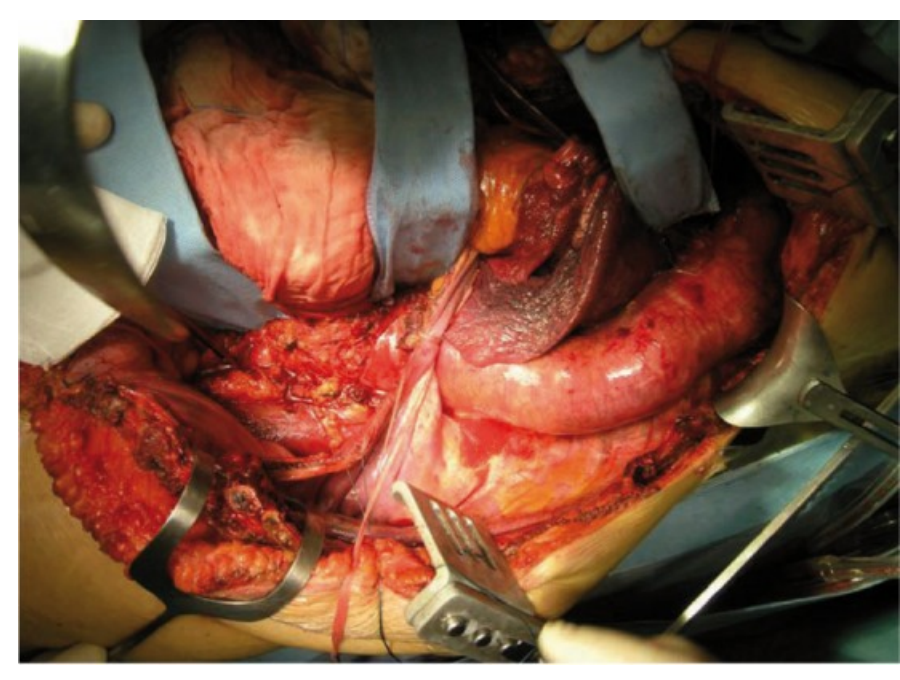

Figure 1. Exposure of thoracoabdominal aortic aneurysm type II through thoracofrenolaparotomy (Chiesa 2009)

center. Under favorable anatomical conditions, a limited frenotomy is performed to preserve the tendon center of the diaphragm; this was shown to reduce the time of respiratory weaning [3].

The aortic segment of the upper abdomen is exposed transperitoneally; retroperitoneum is introduced laterally to the left colon, and medial visceral rotation is performed so that the left colon, spleen, and left kidney can be retracted anteriorly and to the right. The transperitoneal approach allows direct view of the abdominal organs to evaluate the effectiveness of revascularization at the end of aortic repair [4].

*Correspondence to: Marina Alves Jacintho de Mello, Avenue José Munia, 6300- São José do Rio Preto/SP- Brazil; Tel: 5517997884321; E-mail: mazzacamila5@hotmail.com

Key words: thoracoabdominal aortic aneurysm, reconstruction, surgical treatment.

Received: December 05, 2018; Accepted: December 11, 2018; Published: December 14, 2018 


\section{Left Heart Bypass (LHB)}

Clamping of the descending thoracic aorta leads to various hemodynamic disorders, including severe post-load increase and organic ischemia. The logic of LHB is to provide flow to the spinal cord, viscera and kidneys during the period of aortic clamping along with the reduction of proximal and post-load hypertension in the heart. In the preparation for CLB and aortic clamping, intravenous heparin $(1 \mathrm{mg} /$ $\mathrm{kg}$ ) is administered with a target ACT (Activated Coagulation Time) of 220-270 seconds [5].

The proximal descending thoracic aorta, left atrium, or pulmonary vein are usually cannulated for drainage of arterial blood which is reinfused through a centrifugal pump (Biomedicus) into the subdiaphragmatic aorta or the common left femoral artery. The flow is initially low $(500 \mathrm{~mL} / \mathrm{min})$ to prevent retrograde embolization and then increases after aortic clamping to an average distal aortic pressure of about $70 \mathrm{mmHg}$, a value generally achieved using a flow rate of 1500 to $2500 \mathrm{~mL} / \mathrm{min}$. A "Y" bifurcation is connected to the circuit and is provided with two occlusion / perfusion catheters for selective perfusion of visceral vessels (Figure 2) [5].

\section{Aortic repair}

Since the proximal aspect of TAAA is isolated between calipers, the descending thoracic aorta is sectioned and separated from the esophagus (Figure 3).

The proximal end of the graft is sutured to the descending thoracic aorta using a $2 / 0$ monofilament polypropylene suture in a standard manner. The forceps are then removed and reapplied to the abdominal aorta above the celiac axis (sequential clamping).

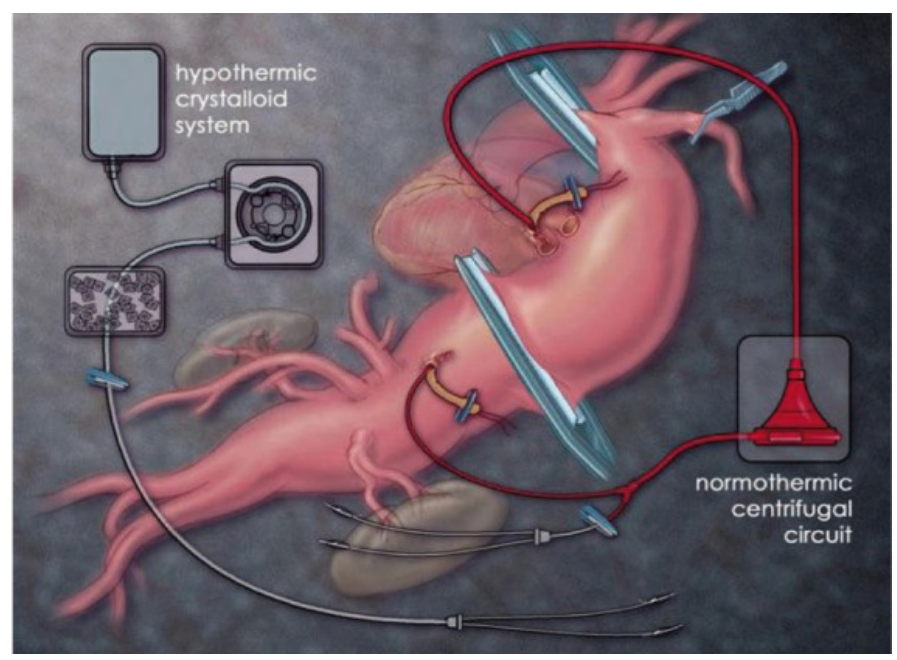

Figure 2. Left heart deviation and renal perfusion catheters (Chiesa 2009)
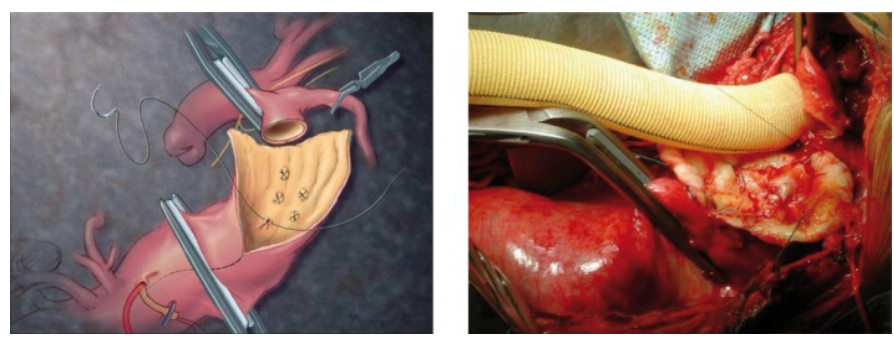

Figure 3. Transection of the thoracic aorta and end-to-end anastomosis. Ligature of the segmental arteries is also shown (Chiesa 2009)
Replantation of intercostal arteries to the aortic graft plays a critical role in the protection of the spinal cord [6]. The critical patent segmental arteries of T7 to L2 are selectively returned to the graft by means of aortic flap or graft interposition. These arteries may be temporarily occluded with catheters to prevent the phenomenon of blood theft [4].

The distal forceps are moved to the distal abdominal aorta below the renal arteries and the upper abdominal aortic aneurysm is opened. The visceral blood perfusion is then maintained by the pump with occlusion / perfusion catheters (9 Fr) selectively inserted into the celiac trunk and the superior mesenteric artery $(400 \mathrm{~mL} /$ $\mathrm{min}$ ). Selective perfusion of the renal arteries is performed with a cold crystalloid solution (Ringer $4^{\circ} \mathrm{C}+$ mannitol $18 \% 70 \mathrm{~mL}$, 6-methylprednisolone $500 \mathrm{mg}$ in $500 \mathrm{~mL}$ ) [5].

For the reimplantation of the visceral arteries, a lateral cut is adapted in the graft and in the celiac trunk, superior mesenteric artery and renal arteries are replaced by means of a Carrel adhesive. This technique was performed in $82.3 \%$ of patients in our series [4].

In $33.1 \%$ of cases treated with Carrel patch, the left renal artery was separately repositioned to the graft either directly or by graft interposition. When the relative distance of the visceral arteries would require a large Carrel patch, a branched graft can be used successfully (Figure 4) [5].

This prosthesis allows the reinsertion of a single vessel, reducing the risk of recurrent aortic aneurysm of the aortic flap. In our series, the Coselli branched graft was used in $10.5 \%$ of the cases [5]. The Vascutek Triplex $^{\text {Tw }}$ graft is a new vascular prosthesis and consists of three layers: an internal polyester graft, an outer layer of ePTFE and a central layer of elastomeric membrane (Figure 5). In our preliminary experience with this graft, we found good performances of handling and adaptation and, indeed, reduction of bleeding of the suture lines [5]. Finally, an end-to-end anastomosis with the distal aorta is performed. In some cases (TAAA type I), the visceral arteries may be incorporated into a bevelled distal anastomosis.

\section{Hybrid repair}

Endovascular procedures may be a less invasive approach to the thoracoabdominal aorta, however, involvement of the visceral segment of the aorta represents a major challenge for repair of the TAAA stent [5].

Although total endovascular treatment with branched endoprosthesis has made visceral perfusion preservation technically feasible, the cost-effectiveness and durability of these pioneering techniques have not yet been fully evaluated [6-8].

The hybrid TAAA repair was first introduced by QuiñonesBaldrich in 1999 and consists mainly of open aortic debranching and revascularization, followed by endovascular exclusion of the aneurysm. The entry site for the visceral grafts is a healthy artery, usually the infra-renal aorta, the iliac arteries, or an infra-renal graft. The visceral and renal arteries are then ligated at the origin to prevent reflux in the aneurysm and consequent type II leakage. The open surgical stage requires a laparotomy and a transperitoneal or extraperitoneal access to the visceral vessels; however, proximal aortic clamping, thoracotomy, aneurysmal exposure and monopulmonary ventilation are avoided (Figure 6) [5].

Surgical and endovascular procedures may be simultaneous or staged.The repair of the hybrid TAAA may be indicated in the case of repair of the anterior descending thoracic aorta, in which a 


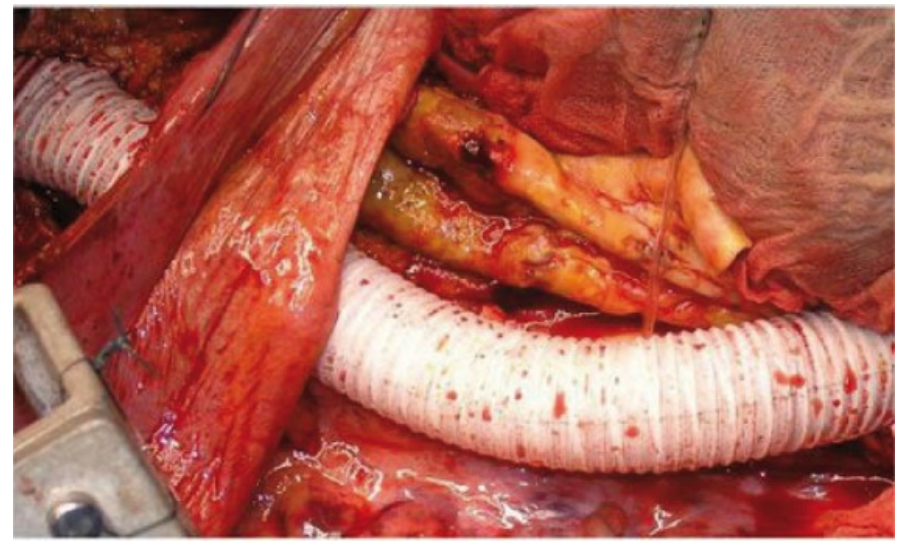

Figure 4. Repair of TAAA type II: replacement of the aortic graft and reinsertion of the visceral vessels by means of the Carrel patch (left) and Coselli's thoracoabdominal graft (above) (Chiesa 2009)

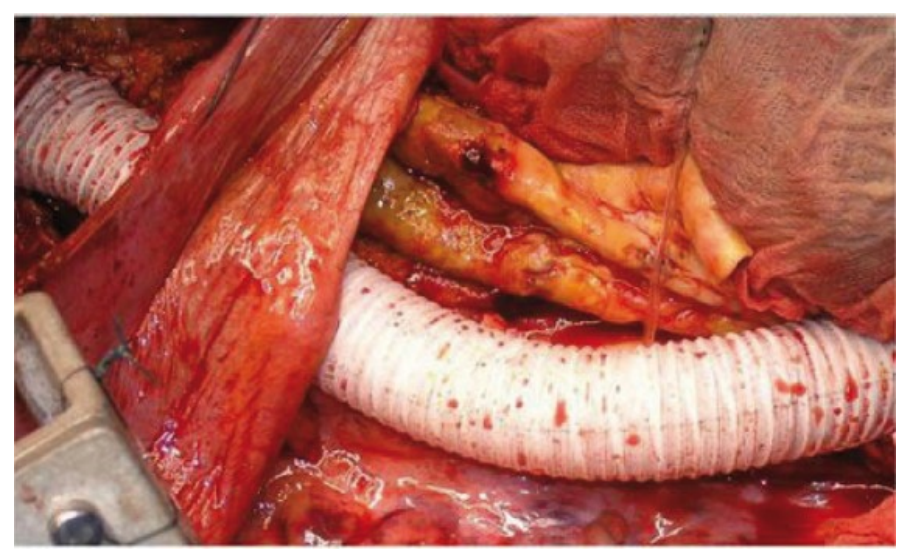

Figure 5. The Vascutek Triplex ${ }^{\mathrm{TM}}$ graft consists of three layers: an internal polyester graft, an outer layer of ePTFE and a central layer of elastomeric membrane (Chiesa 2009)

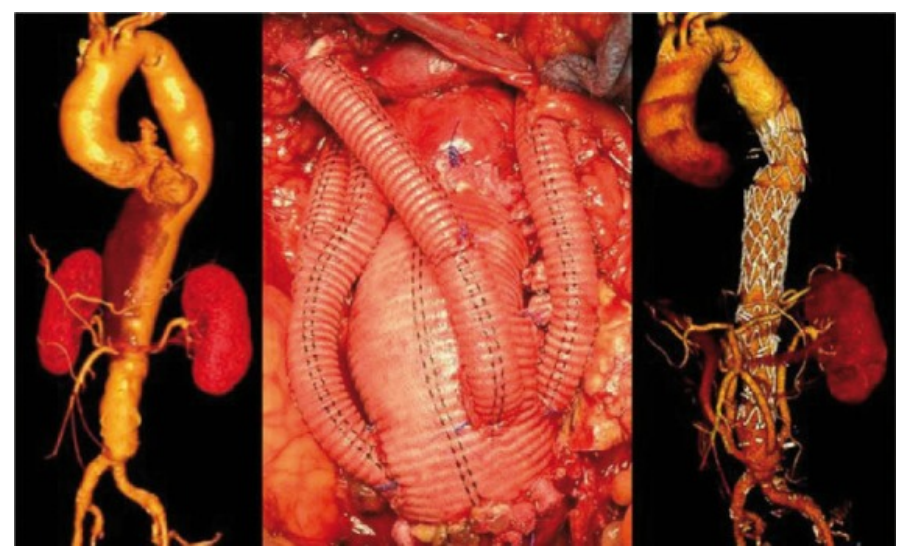

Figure 6. Preoperative CT scan of a patient with TATA type III (left). The hybrid procedure consisted of infra-renal aortic graft with revascularization of single visceral vessels (center) (Chiesa 2009)

thoracotomy to redo on the left side may be associated with greater bleeding, an increase in respiratory and organic insufficiency rate in the postoperative period and a longer total time of aortic clamping [6].

Another advantage of the hybrid treatment is the possibility of reducing organ ischemia time and performing visceral protection techniques through selective cooling [6]. The hybrid repair is attractive in the case of visceral aortic patch aneurysm (VAP) after the conventional TAAA repair.
In addition, PAV aneurysms have ideal proximal and distal straight and long neck, where the endoprosthesis can be safely administered. With this technique, the branches of the aorta are anastomosed separately and virtually no remnant of the native aorta is left in situ, thus avoiding the risk of recurrences.

\section{Surgical repair of thoracoabdominal aortic aneurysms by the technique of critical reinsertion of the artery}

Thoracoabdominal aortic aneurysm refers to aneurysms of the descending thoracic or infra-renal abdominal aorta. The most serious complications of aortic aneurysms are dissection and rupture of the aorta, which often result in death of the patient. With an aging population and improved diagnostic capabilities, more and more patients are increasingly being referred for the treatment of thoracoabdominal aortic aneurysm. Conventional open repair of thoracoabdominal aneurysms and dissections remains complex and demanding and is associated with significant morbidity and mortality. Improving the therapeutic effect of surgery requires continuous improvement of surgical methods. In the present study, we sought to retrospectively analyze the efficacy and safety of surgical treatment in 23 cases of thoracoabdominal aortic aneurysm using sequential aortic clamping and critical arterial reinsertion [7].

The natural history of thoracoabdominal aortic aneurysm shows a poor prognosis, as illustrated by the high rates of dissection in non-operated patients. In addition, population aging and improved diagnostic capabilities have led to a substantial increase in surgical demand for patients with thoracoabdominal aortic aneurysm. However, open surgical treatment of thoracoabdominal aortic aneurysm continues to be associated with significant mortality, and there is also a significant risk of visceral, renal, and spinal ischemia. To reduce the considerable operative morbimortality, the researchers elaborated many strategies to substantially improve the surgical outcome of the thoracoabdominal aortic aneurysm. With the application of new strategies to further reduce mortality and prevent end-organ ischemia associated with thoracoabdominal aortic aneurysm, surgical repair is widely considered as a first option for thoracoabdominal aortic aneurysm [2].

Despite the surgical advances, patients with thoracoabdominal aortic aneurysm remain at high risk for the development of postoperative complications. Before the operation, the risk of rupture should be weighed against the risk of an adverse surgical outcome. The careful selection of patients for open surgical repair is based on an extensive surgical risk assessment. The continuous improvement of perioperative management of patients and the application of new surgical techniques is essential to reduce the incidence of complications [8].

Preoperative management of patients undergoing thoracoabdominal aortic aneurysm repair has evolved substantially during the last two decades. Several authors reported excellent results using different protection strategies, such as motor evoked potential monitoring, epidural cooling, and hypothermic circulatory arrest. Our application of the technique of critical arterial reinsertion and sequential clamping in the surgical therapy of thoracoabdominal aortic aneurysm provides excellent results. The surgical cure rate was $95.65 \%$. Routine surgical modalities include the use of moderate systemic heparinization, moderate permissive hypothermia $\left(32^{\circ}\right.$ $\mathrm{C}$ to $34^{\circ} \mathrm{C}$ ), and sequential aortic clamping. Our current strategy to prevent organ damage in elective patients is to use distal aortic perfusion (maintaining distal perfusion pressure equal to or greater than $70 \mathrm{mmHg}$ ) in combination with liberal replacement of critical 
arteries, drainage of cerebrospinal fluid, cold crystalloid renal perfusion and selective visceral perfusion. This multimodal approach is based on findings from other surgeons with extensive experience and is also supported by our own results [6].

In the sequential clamping technique, as the aorta is replaced from the proximal to the distal portion of the lesion, the aortic clamping is moved sequentially to lower positions along the graft to restore perfusion in newly relocated branches. Sequential clamping of the aorta may reduce the time of ischemia and improve perfusion of the abdominal viscera. In theory, reinsertion of critical arteries should prevent spinal cord injury by preserving circulation to the anterior spinal artery; however, the question of which specific arteries to connect and which ones to relocate remains in dispute. Considering that the spinal cord has redundant blood supply according to collateral network theory and questioned the utility of critical artery replacement, we and others believe that because of the anatomical complexities of the medullary circulation and the commonly incomplete anterior spinal artery In addition to the variations and aggressive reinsertion strategies, especially those between $\mathrm{T} 8$ and L2, can prevent problems in the spinal cord. Although clinically significant post-operative manifestations of hepatic, pancreatic and intestinal ischemia are infrequent, they can have a devastating impact when they occur. To reduce the risk of perioperative coagulopathy and bacterial translocation, after the aorta was opened adjacent to the visceral branches, we used separate balloon perfusion catheters to selectively perfuse the visceral arteries. Passive hypothermia was used in all cases. We intentionally allowed the body temperature to drop to $32-34^{\circ} \mathrm{C}$ during the procedure. Hypothermia, by decreasing the metabolic demands of the spinal cord, may be beneficial in the prevention of postoperative paraplegia. After completion of the operation, patients were reheated at $37^{\circ} \mathrm{C}$. Our experience with CSF drainage during thoracoabdominal aortic aneurysm repair is that the amount of CSF drained is dependent on CSF pressure, which should be maintained below $10 \mathrm{mmHg}$. Postoperative renal dysfunction is another major concern, so we carefully monitor urinary output and serum creatinine levels throughout the postoperative period. Intravenous antibiotics are maintained until all drains, thoracic tubes and central venous lines are removed [7].

In summary, this experience shows that the use of critical aortic clamping and technique of segmental artery reinsertion could significantly improve the cure rate and reduce the morbidity of postoperative complications, including paraplegia, abdominal viscera ischemia and renal insufficiency [9].

\section{Surgical treatment of thoracoabdominal aortic aneurysm after correction of thoracic or infra-renal abdominal aortic aneurysm}

The long-term results of most thoracic or abdominal aortic aneurysm reconstructions are satisfactory. However, some patients require a reoperation for a separate aneurysm after the original aortic reconstruction. Thus, in cases of thoracic or abdominal aortic aneurysms, there is a fairly high risk of developing multiple aortic aneurysms. Studies have reported that $59.6 \%$ of patients who originally presented aortic aneurysms involving the ascending, transverse or descending artery segments developed multiple aneurysms, whereas multiple aneurysms developed in only $12 \%$ of patients who initially had abdominal aortic aneurysms. Studies have reported that new or recurrent aortic aneurysms were responsible for 36 of 130 (27.7\%) reoperations of the thoracic aorta in 120 patients. Most reports on surgical treatment of recurrent and multiple aneurysms present results of more proximal aortic operations after repair of infra-renal abdominal aortic aneurysm. Mortality rates in previous reports range from $12.2 \%$ to $28.6 \%$ [1].

There are few reports on the repair of thoracoabdominal aortic aneurysm (TAAA) in patients with previous thoracic aortic aneurysm repair. Of the 1509 patients in the complete Crawford TAAA trial, reported by Svensson et al., 181 (12\%) had been previously operated on proximal aortic, and this group of patients was characterized by a $30-$ day lower mortality rate compared to the group of patients ( $4 \%$ in the group with previous correction of thoracic aortic aneurysm versus $9 \%$ in the group with no previous correction of thoracic aortic aneurysm, $\mathrm{P}$ $=.0025$ ) were not previously submitted to repair of the thoracic aortic aneurysm. In addition, Coselli and colleagues reported that prior repair of thoracic aortic aneurysm did not adversely affect the outcome of thoracoabdominal aortic aneurysm repair [10].

The reported results of surgical repair of multilevel and recurrent aortic aneurysms vary widely. Therefore, the safety of TAAA repair in patients with previous thoracic or abdominal aortic aneurysm repair remains uncertain. Since 1982, we have used visceral and renal selective perfusion during thoracoabdominal aortic repair. Studies have retrospectively evaluated the experience with TAAA repair to compare outcomes in patients who underwent and those who had not undergone thoracic or infra-renal descending abdominal aortic aneurysm [11].

In contrast to patients who had undergone thoracic or infrarenal abdominal aortic aneurysm repair (group I), those who had not previously undergone thoracic or infra-renal aortic aneurysm repair (group II) were and did not present a significant difference co-morbid disease. The highest proportion of patients with Marfan syndrome in the first group undoubtedly contributed to these age differences and coexisting disease. The longer aneurysms and the higher prevalence of dissection in group I explain the longer distal aortic perfusion times. Although less extensive aneurysms and shorter clamp and perfusion times, 30-day mortality and in-hospital mortality rates in group II patients tended to be higher. However, there was no significant difference in mortality at 30 days and in in-hospital mortality rates between the two groups. An intrinsic selection bias for group I may have contributed to the lower 30-day mortality rate in this group. Careful medical follow-up after the initial operation may have resulted in the prevention of risk factors. In addition, as some patients who would need future correction of TAAA did not survive the initial aortic operation, several patients with significant comorbid disease may have been automatically eliminated from group I. Therefore, surviving the previous operation, the group of patients already had been submitted to correction of thoracic or infra-renal descending abdominal aortic aneurysm may have been inherently more likely to be able to tolerate TAAA repair compared to those who had undergone no downward repair of the thoracic or infra-renal abdominal aorta [12].

There is no significant difference between complication rates or mortality rates in the two groups. In group I patients, there was a greater risk of lung injury during thoracotomy due to pulmonary adhesion, and it was believed that there would be a higher incidence of postoperative respiratory complications in this group, but a significant difference between the incidence of pulmonary complications in the group. two groups were not found.

More patients previously submitted to thoracic or descending infrarrenal aortic aneurysm repair (Group I) had Marfan's syndrome than patients who had not previously performed repair of the aneurysm (Group II) and an increase in chronic dissection was responsible for 
$64 \%$ of the causes of TAAA in group I $(P=0.003)$. However, the fact that there is no significant difference between the complications in the two groups suggests that chronic dissection has no effect on the likelihood of paraplegia or other postoperative complications occurring. Studies have reported that chronic dissociation of the aorta is not a risk factor for postoperative paraplegia in cases of TAAA. Although no significant differences were found between the incidences of postoperative paraplegia in the two groups in the present study, a high incidence of paraplegia was found in patients who had already undergone thoracic or infra-renal abdominal aortic aneurysm repair. However, Coselli et al. reported a low rate of paraplegia in patients who had already undergone thoracic or infra-renal abdominal aortic aneurysm repair (SANTOS, 2004).

Many recent studies have shown that CSF drainage is useful for the protection of the spine. Cerebrospinal fluid drainage has been used since 1999, but we have not yet accumulated enough data from the cases to conclude whether CSF drainage is effective or not. We were also able to identify the artery of Adamkiewicz in the ARM, and identification of this artery provided useful preoperative information. The artery of Adamkiewicz is identifiable in approximately $70 \%$ of the patients, and the identification of this artery provides useful information for the reconstruction of the intercostal arteries and lumbar artery in the TAAA, besides allowing the time of surgery to be reduced [10].

Deep hypothermic circulatory arrest in TAAA repair was used in eight of the patients in the present study after 1995 (four patients in each group). These results suggest that TAAA repairs using deep hypothermic circulatory arrest cannot be performed with generally acceptable rates of mortality and low morbidity. The role of deep hypothermic circulatory arrest during distal aortic surgery remains controversial. Several recent studies have shown that there are associated high rates of mortality and morbidity. Safi et al., For example, reported that six of the 21 patients who underwent hypothermic circulatory arrest due to failure to achieve proximal aortic control died within 30 days (28.6\%). Excluding two intraoperative deaths, paraplegia developed in $10.5 \%$ of the patients and stroke occurred in $31.6 \%$ of the patients. Studies report that the main disadvantages of the technique of deep hypothermia through left thoracotomy are lung injury secondary to manipulation of the left lung and disorders of blood coagulation. Considering the high rates of hospital mortality and postoperative complications, it seems that deep hypothermia should be limited to patients in whom segmental aortic clamping cannot be performed [13].

\section{Conclusion}

The long-term results of most thoracic or abdominal aortic aneurysm reconstructions are satisfactory. However, some patients require a reoperation for a separate aneurysm after the original aortic reconstruction.

In conclusion, the presence of a thoracic or infra-renal descending abdominal aortic aneurysm does not negatively affect the result of the TAAA repair. Therefore, we recommend at least one semester computed tomography scan of the chest and abdomen for follow-up in patients who have had an initial repair of the aortic aneurysm.

\section{References}

1. NETO, Mioto (2005) Treatment of aortic lesions in closed thoracic injuries. $J$ Vascular.

2. ROLIM, Delilah (2016) Thoraco-abdominal aneurysm post-chronic type B dissection an anatomical challenge with an unexpectedly simple endovascular solution. Angiology and Vascular Sur.

3. Rodrigo de Castro Bernardes, Fernando Antônio Roquette Reis Filho, Atena Cipriano Castro, Walter Rabelo, Marcos Antônio Marino, et al. (1994) Surgical correction of aortic aneurysms: a new device that transforms any type of prosthesis into an intraluminal prosthesis. Rev Bras Cir Cardiovasc 9: 54-59.

4. Belczak (2010) Endovascular treatment of aortic aneurysms in patients with Behçet's disease: report of two cases. Vascular $j$.

5. Chiesa R, Civilini E, Melissano G, Logaldo D, Calliari FM, et al. (2009) Management of thoracoabdominal aortic aneurysms. HSR Proc Intensive Care Cardiovasc Anesth 1: 45-53. [Crossref]

6. Fantini FA (2009) Surgical treatment of aneurysms and aortic arch dissections. Revis Bras Ciru.

7. Rock EF (2004) Broken thoracoabdominal aneurysm: modification of the visceral perfusion circuit. Rev bras Cir.

8. Berlinck MF (1990) Surgical treatment of aortic dissection. Revis bras Cir.

9. Albuquerque LC (2009) Guidelines for the surgical treatment of aortic diseases of the Brazilian Society of Cardiovascular Surgery: update. Revista de Cirurgia.

10. Saadi EK (2006) Endovascular treatment of abdominal aortic aneurysms: initia experience and results in the short and medium term. Brazilian Society of Surgery.

11. Santos VP (2004) Broken thoracoabdominal aneurysm: report of a case with the use of an intraluminal grooved rigid ring in the proximal anastomosis. $J$ Vascular.

12. Rojas SS, Veiga VC, Souza JM, Berlinck MF, Senra DF, et al. (2005) [Correction of thoracic and thoracoabdominal aortic aneurysms. Central cannulation technique]. Arq Bras Cardiol 84: 297-303. [Crossref]

13. Albuquerque LC, Palma JH, Braile D, Gomes W, Guimarães JI (2004) [Guidelines for surgery in aortic disease]. Arq Bras Cardiol 82 Suppl 5: 35-50. [Crossref]

Copyright: (C2018 Jacintho de Mello MA. This is an open-access article distributed under the terms of the Creative Commons Attribution License, which permits unrestricted use, distribution, and reproduction in any medium, provided the original author and source are credited. 